\title{
CONGRUENCE PERMUTABILITY FOR ALGEBRAS WITH PSEUDOCOMPLEMENTATION
}

\author{
by R. BEAZER \\ (Received 19th October 1979)
}

\section{Introduction}

In this note we are concerned with the permutability of congruence relations on semilattices and lattices with pseudocomplementation. There are some results in the literature along these lines. For example, in (8) H. P. Sankappanavar characterises those pseudocomplemented semilattices whose congruence lattice is modular and employs the result in conjunction with the well-known fact that algebras with permuting congruences are congruence-modular to characterise those pseudocomplemented semilattices with permuting congruences. Our first result is a direct, short proof of his result. In (2), J. Berman shows that for all congruences on a distributive lattice $L$ with pseudocomplementation to permute it is necessary and sufficient that $D(L)$, the dense filter of $L$, be relatively complemented. Our second result is a generalisation of that result to an important equational class of lattices with pseudocomplementation which properly contains the modular lattices with pseudocomplementation.

\section{Preliminaries}

A p-semilattice (pseudocomplemented semilattice) is an algebra $\left\langle S ; \wedge,{ }^{*}, 0\right\rangle$ in which the deletion of the unary operation ${ }^{*}$ yields a meet semilattice with 0 and $a^{*}$ is characterized by $a \wedge x=0$ if and only if $x \leqq a^{*}$. If, for any $p$-semilattice $S$, we write $B(S)$ for $\left\{x \in S ; x=x^{* *}\right\}$ (the skeleton of $S$ ) and $D(S)$ for $\left\{x \in S ; x^{* *}=1\right\}$ (the set of dense elements of $S)$ then $\langle B(S) ; \cup, \wedge, *, 0,1\rangle$ is a Boolean algebra when $a \cup b$ is defined to be $\left(a^{*} \wedge b^{*}\right)^{*}$, for any $a, b \in B(S)$, and $D(S)$ is a filter in $S$. A (modular, distributive) $p$-algebra (lattice with pseudocomplementation) is an algebra $-\langle L ; \vee$, $\wedge, *, 0,1\rangle$ in which the deletion of ${ }^{*}$ yields a bounded (modular, distributive) lattice and * is the operation of pseudocomplementation. A p-algebra is said to be quasi-modular if it satisfies the identity

$$
\left[(x \wedge y) \vee z^{* *}\right] \wedge x=(x \wedge y) \vee\left(z^{* *} \wedge x\right) .
$$

This class of $p$-algebras was introduced by $T$. Katrinák in (7) and.is of some importance in that it properly contains the class of modular $p$-algebras and shares with it sufficiently many important properties that T. Katriňák was able in (6) to extend to this class his description of the subdirectly irreducible modular p-algebras (see (6)). By a congruence relation on a $p$-semilattice ( $p$-algebra) we mean a semilattice (lattice) 
congruence preserving $*$. The relation $\phi$ defined on a $p$-semilattice or a $p$-algebra by $a \equiv b(\phi)$ if and only if $a^{*}=b^{*}$ is known to be a congruence, called the Glivenko congruence. An algebra $A$ is said to have permuting congruences whenever the usual relational product $\theta \circ \psi$ of any pair of congruences $\theta, \psi$ on $A$ commutes; that is, $a \equiv t(\theta)$ and $t \equiv b(\psi)$ implies $a \equiv w(\psi)$ and $w \equiv b(\theta)$, for some $w \in A$. If $\theta$ is a congruence on $A$ and $S \subseteq A$ then we denote by $\theta \mid S$ the restriction of $\theta$ to $S$.

We refer to (4) not only for the standard results about $p$-semilattices and $p$-algebras (see also (3)) but also for general lattice-theoretic notation, terminology and results. In particular, $a / b$ will denote an ordered pair of elements satisfying $b \leqq a$ in a (semi) lattice $L$ and will be called a quotient of $L$. If for a pair of quotients $a / b, c / d$ in a lattice $L$ there is a sequence $c / d=e_{0} / f_{0}, e_{1} / f_{1}, \ldots, e_{n} / f_{n}=a / b$ of quotients $e_{i} / f_{i}$ satisfying $e_{i+1} \vee f_{i}=e_{i}$ and $e_{i+1} \wedge f_{i} \geqq f_{i+1}$, or $f_{i+1} \wedge e_{i}=f_{i}$ and $f_{i+1} \vee e_{i} \leqq e_{i+1}(0 \leqq i \leqq n-1)$ then we write $c / d \approx_{w} a / b$ and say that $c / d$ is weakly projective into $a / b$ in $L$. If $a \geqq x, y \geqq b$ then $y$ is said to be a weak relative complement of $x$ in $[a, b]$ if there is a chain $a=e_{0} \geqq e_{1} \geqq$ $\cdots \geqq e_{m}=y=f_{0} \geqq f_{1} \geqq \cdots \geqq f_{n}=b$ such that each $e_{i} / e_{i+1} \approx_{w} x / b$ and each $f_{j} / f_{i+1} \approx_{w} a / x$. A lattice $L$ is said to be relatively complemented in the weak sense if every element in any interval of $L$ has a weak relative complement. Hashimoto (5) has shown that a lattice has permuting congruences if and only if it is relatively complemented in the weak sense.

\section{Permuting Congruences}

A simple proof of our first lemma can be found in (1).

Lemma 1. If $S$ is a p-semilattice, $a / b$ is a quotient of $S$ and $\theta(a, b)$ denotes the smallest (principal) congruence on $S$ collapsing $a / b$ then

(i) $x \equiv y(\theta(1, a))$ if and only if $x \wedge a=y \wedge a$.

(ii) If $a^{*}=b^{*}$ then $x \equiv y(\theta(a, b))$ if and only if $x=y$, or $x, y \leqq a$ and $x \wedge b=y \wedge b$.

Theorem 2. A p-semilattice has permuting congruences if and only if it is a Boolean algebra or a Boolean algebra with a new unit adjoined.

Proof. Let $S$ be a $p$-semilattice having permuting congruences. If $a \in S$ then there exists $t \in S$ such that $a \equiv t\left(\theta\left(1, a^{* *}\right)\right)$ and $t \equiv 1\left(\theta\left(a^{* *}, a\right)\right)$, since $a \equiv$ $1\left(\theta\left(a^{* *}, a\right) \circ \theta\left(1, a^{* *}\right)\right)$. An application of Lemma 1(i) to $a \equiv t\left(\theta\left(1, a^{* *}\right)\right)$ yields $a=$ $a \wedge a^{* *}=t \wedge a^{* *}$. Furthermore, since $a^{*}=a^{* * *}$, an application of Lemma 1(ii) to $t \equiv 1\left(\theta\left(a^{* *}, a\right)\right)$ shows that $t=1$ or $a^{* *}=1$. We conclude that either $a=a^{* *}$ or $a^{* *}=1$. Consequently, $S=B(S) \cup D(S)$. Next, we claim that $D(S)$ contains at most one element distinct from 1 . If not, then $D(S)$ contains a three-element chain, say $1>a>b$, so that, since $b \equiv 1(\theta(a, b) \circ \theta(1, a))$ and $S$ has permuting congruences, there exists $t \in S$ such that $b \equiv t(\theta(1, a))$ and $t \equiv 1(\theta(a, b))$. An application of Lemma 1(i) to $b \equiv t(\theta(1, a))$ yields $b=t \wedge a$. Furthermore, since $a^{*}=b^{*}=0$, we can apply Lemma 1(ii) to $t \equiv 1(\theta(a, b))$ and infer that $t=1$ or $a=1$. We conclude that $a=b$ or $a=1$ and, in any case, have a contradiction. Of course, if $D(S)=\{1\}$ then $S$ concurs with the Boolean algebra $B(S)$. If $D(S)$ contains exactly one element $d$ distinct from 1 , so that $S=B(S) \cup\{d\}$, and $x \in B(S) \backslash\{1\}$ then $x \wedge d=(x \wedge d)^{* *}=x^{* *} \wedge d^{* *}=x$ from which it 
follows that $x \leqq d$, for any $x \in S \backslash\{1\}$. Furthermore, the interval $[0, d]$ in $S$ is a Boolean algebra. Indeed, $[0, d]$ is a $p$-semilattice in which the pseudocomplement $x^{\circledast}$ of $x$ in $[0, d]$ is $x^{*} \wedge d$. Moreover, if $x \in[0, d]$ then $x^{\circledast \otimes}=\left(x^{*} \wedge d\right)^{*} \wedge d=\left(x^{* * *} \wedge d^{* *}\right)^{*} \wedge d=$ $x^{* *} \wedge d=x$, since $x=x^{* *}$ or $x=d$, and so $[0, d]$ concurs with its skeleton $B([0, d])$. Thus, $S$ is a Boolean algebra with a new unit adjoined.

The converse is an easy consequence of the well-known fact that any Boolean algebra has permuting congruences.

The constituents of the following lemma may be found in (7).

Lemma 3. Let $L$ be a quasi-modular p-algebra. Then

(i) $L$ satisfies the identity $x=x^{* *} \wedge\left(x \vee x^{*}\right)$.

(ii) If $a / b, c / d$ are quotients of $D(L)$ and $a / b \approx_{w} c / d$ in $L$ then $a / b \approx_{w} c / d$ in $D(L)$.

Theorem 4. Let $L$ be a quasi-modular p-algebra. Then the following are equivalent:

(i) $L$ has permuting congruences,

(ii) $D(L)$ has permuting congruences,

(iii) $D(L)$ is relatively complemented in the weak sense.

Proof. Let $L$ have permuting congruences. In order to show that the (lattice) congruences on $D(L)$ permute we only have to show that any pair of principal (lattice) congruences on $D(L)$ permute. Indeed, if $\theta, \psi$ are congruences on $D(L), \theta_{D}(d, e)$ denotes the principal congruence on $D(L)$ collapsing the quotient $d / e$ of $D(L)$ and $x \equiv t(\theta), \quad t \equiv y(\psi)$ for some $t \in D(L)$ then $x \equiv y\left(\theta_{\mathrm{D}}(t, y) \circ \theta_{\mathrm{D}}(x, t)\right)$, since $x \equiv$ $y\left(\theta_{D}(x, t) \circ \theta_{D}(t, y)\right)$ and we are assuming that $\theta_{D}(x, t), \theta_{D}(t, y)$ permute. Therefore, there exists $w \in D(L)$ such that $x \equiv w\left(\theta_{D}(t, y)\right)$ and $w \equiv y\left(\theta_{D}(x, t)\right)$ from which it follows that $x \equiv y(\psi \circ \theta)$, since $\theta_{D}(x, t) \leqq \theta$ and $\theta_{D}(t, y) \leqq \psi$. Next, observe that if $\theta_{\text {Lat }}(d, e)$ denotes the principal lattice congruence on $L$ collapsing a quotient $d / e$ of $D(L)$ then $\theta_{D}(d, e)=\theta_{\text {Lat }}(d, e) \mid D(L)$ is an immediate consequence of Lemma 3(ii) and the wellknown description of principal lattice congruences in terms of weak projectivities (see [4], Chap 3, Theorem 1.2). Furthermore, notice that $\theta_{\text {Lat }}(d, e)$ coincides with $\theta(d, e)$ the smallest congruence on $L$ collapsing the quotient $d / e$ of $D(L)$. Indeed, $\theta_{\text {Lat }}(d, e) \leqq \phi$, since $d^{*}=e^{*}(=0)$, so that $\theta_{\text {Lat }}(d, e)$ preserves $*$ and, therefore, $\theta(d, e) \leqq$ $\theta_{\text {Lat }}(d, e)$. It follows now, that $\theta_{D}(d, e) \circ \theta_{D}(f, g)=\theta(d, e)|D(L) \circ \theta(f, g)| D(L)$ for any pair of quotients $d / e, f / g$ of $D(L)$. However, $\theta(d, e) \mid D(L)$ 。 $\theta(f, g)|D(L)=(\theta(d, e) \circ \theta(f, g))| D(L)$. Indeed, if $x, y \in D(L)$ and there exists $t \in L$ such that $x \equiv t(\theta(d, e))$ and $t \equiv y(\theta(f, g))$ then, since $x^{*}=y^{*}=0, t^{*} \equiv 0(\theta(d, e) \cap$ $\theta(f, g))$ so that $x \equiv t \vee t^{*}(\theta(d, e))$ and $t \vee t^{*} \equiv y(\theta(f, g))$ from which it follows that $x \equiv y(\theta(d, e)|D(L) \circ \theta(f, g)| D(L))$. Therefore, $\quad(\theta(d, e) \circ \theta(f, g)) \mid D(L) \leqq$ $\theta(d, e)|D(L) \circ \theta(f, g)| D(L)$; the reverse inclusion being obvious. In conclusion, $\theta_{D}(d, e) \circ \theta_{D}(f, g)=(\theta(d, e) \circ \theta(f, g)) \mid D(L)$ and so $\theta_{D}(d, e) \circ \theta_{D}(f, g)=\theta_{D}(f, g) \circ \theta_{D}(d, e)$ is a consequence of the permutability of the corresponding principal congruences on $L$.

Conversely, suppose that $D(L)$ has permuting congruences and let $\theta, \psi$ be congruences on $L$. First, observe that in order to show that $x \equiv y(\psi \circ \theta)$ it is sufficient to show that $x^{* *} \equiv y^{* *}(\psi \circ \theta)$ and $x \vee x^{*} \equiv y \vee y^{*}(\psi \circ \theta)$. Indeed, if there exists $p, q \in L$ such that $x^{* *} \equiv p(\psi), p \equiv y^{* *}(\theta)$ and $x \vee x^{*} \equiv q(\psi), q \equiv y \vee y^{*}(\theta)$ then, by Lemma 3(i), $x=$ $x^{* *} \wedge\left(x \vee x^{*}\right) \equiv p \wedge q(\psi)$ and $y=y^{* *} \wedge\left(y \vee y^{*}\right) \equiv p \wedge q(\theta)$ so that $x \equiv y(\psi \circ \theta)$. With this 
in mind, let $x \equiv t(\theta)$ and $t \equiv y(\psi)$. Then $x^{* *} \equiv t^{* *}(\theta)$ and $t^{* *} \equiv y^{* *}(\psi)$ so that $x^{* *} \equiv$ $t^{* *}(\theta \mid B(L))$ and $t^{* *} \equiv y^{* *}(\psi \mid B(L))$. It follows, since $\theta|B(L), \psi| B(L)$ are easily seen to be congruences in the Boolean algebra $B(L)$ and every Boolean algebra has permuting congruences, that $x^{* *} \equiv y^{* *}(\psi|B(L) \circ \theta| B(L))$ and, therefore, $x^{* *} \equiv$ $y^{* *}(\psi \circ \theta)$. In addition, since $x \equiv t(\theta)$ and $t \equiv y(\psi)$ implies $x \vee x^{*} \equiv t \vee t^{*}(\theta)$ and $t \vee t^{*} \equiv y \vee y^{*}(\psi)$, we have $x \vee x^{*} \equiv y \vee y^{*}(\theta|D(L) \circ \psi| D(L))$ from which we infer $x \vee x^{*} \equiv y \vee y^{*}(\psi|D(L) \circ \theta| D(L))$, since $D(L)$ has permuting congruences. Therefore, $x \vee x^{*} \equiv y \vee y^{*}(\psi \circ \theta)$.

Corollary 5. A distributive p-algebra $L$ has permuting congruences if and only if $D(L)$ is relatively complemented.

Proof. Immediate from Theorem 4 and the well-known fact that a distributive lattice has permuting congruences if and only if it is relatively complemented.

Concluding Remark. Any quasi-modular $p$-algebra satisfies the identity $x=$ $x^{* *} \wedge\left(x \vee x^{*}\right)$ and only this is needed in proving that (ii) implies (i) in Theorem 4. It would be of some interest to know if Theorem 4 holds for the equational class of all $p$-algebras satisfying the identity $x=x^{* *} \wedge\left(x \vee x^{*}\right)$.

\section{REFERENCES}

(1) R. BEAzER, Subdirectly irreducibles for various pseudocomplemented algebras, Algebra Univ. 10 (1980), 225-231.

(2) J. Berman, Congruence relations of pseudocomplemented distributive lattices, Algebra Univ. 3 (1973), 288-293.

(3) G. Grätzer, Lattice theory: First concepts and distributive lattices (Freeman, San Francisco, 1971).

(4) G. Grätzer, General lattice theory (Birkhäuser Verlag, Basel, 1978).

(5) J. HASHIMOTo, Direct, subdirect decompositions and congruence relations, Osaka Math. Jour. 9 (1957), 87-112.

(6) T. KATRIÑÁá, Subdirectly irreducible modular p-algebras, Algebra Univ. 2 (1972), 166173.

(7) T. KAtriñÁá, Subdirectly irreducible p-algebras, Algebra Univ. 9 (1979), 116-126.

(8) H. P. Sankappanavar, A study of congruence lattices of pseudocomplemented semilattices (Ph.D. Thesis, University of Waterloo, 1974).

Department of Mathematics

UNIVERSITY OF GLASGOW

GLASGOW G12 8QW

SCOTLAND 